\title{
THE INVESTIGATION OF THE TRANSIENT REGIMES \\ IN THE NONLINEAR SYSTEMS BY THE GENERALIZED \\ CLASSICAL METHOD
}

\author{
T. ABBASOV AND A. R. BAHADIR
}

Received 10 November 2004 and in revised form 7 January 2005

This paper presents the use of the generalized classical method (GCM) for solving linear and nonlinear differential equations. This method is based on the differential transformation (DT) technique. In the GCM, the solution of the nonlinear transient regimes in the physical processes can be written as a functional series with unknown coefficients. The series can be chosen to satisfy the initial and boundary conditions which represent the properties of the physical process. The unknown coefficients of the series are determined from the differential transformation of the nonlinear differential equation of the system. Therefore, the approximate solution of the nonlinear differential equation can be obtained as a closed-form series.

The validity and efficiency of the GCM is shown using some transient regime problems in the electromechanics processes. The numerical results obtained by the present method are compared with the analytical solutions of the equations. It is shown that the results are found to be in good agreement with each other.

\section{Introduction}

The transient regimes in the electromechanics, mechanics, heat-mass transfer, and hydromechanics are formulated usually by nonlinear differential equations. In many cases, to solve these equations analytically is very difficult or even impossible. Therefore, a large number of numerical techniques have been developed to solve these engineering problems [7]. The most widely used methods are Lindstedt-Poincaré method, the multiple time-scale method, Krylov-Bogoliubov-Mitropolski method, the polynomial series method, Rayleigh-Ritz method, Galerkin method, and so forth [19]. These methods are effective in the solution of the weakly nonlinear systems. However, some difficulties arise in the solution of the strongly nonlinear problems. On the other hand, it is very important to obtain an analytic or a numerical solution of these equations for modeling, optimization, and control of engineering systems.

The integral transform methods such as the Laplace and Fourier transform methods can be used in the solution of the engineering problems [29]. The merit of the integral transform is its ability to transform differential equations to algebraic equations, which 
leads to a systematic and simple solution procedure in the linear problems. However, some difficulties appear in the solution of the nonlinear systems.

Consequently, to develop the effective mathematical methods for solving linear and nonlinear equations analytically or numerically in a wide variety of engineering areas is a current research problem.

Differential transformation (DT) method which is a relatively new method to solve linear and nonlinear equations has received increasing attention in recent years. The ability to obtain both numerical (spectrum) and analytic (power series or functional series) solutions of the differential equations is one of the most important advantages of the DT method. DT method was introduced first by the Ukrainian scientist Pukhov $[20,21,22,23,24,25,26,27,28]$. About 30 years ago, Pukhov gave the fundamentals of the DT method. He obtained the T-transformation of the elementary functions and applied this method to solve the problems of the electrotechnics and electronics [20].

Later, he systematized the differential transformations of the equations and functions [21] and used to solve the linear and the nonlinear equations which appear in the studying transient regimes in the electrical circuits [22]. He also showed that the DT method is effective to solve linear and nonlinear ODEs and PDEs with initial and boundary conditions in the modeling physical systems [23, 24, 25, 26, 27, 28].

During the last ten years, considerable attention has been focused on the application of the DT method $[1,2,3,4,5,6,8,9,10,11,12,13,14,15,16,17]$. In these studies, some new formulas and the correlations are developed to apply the DT method for solving the multidimensional PDEs $[1,4,5,6,9,15,17]$.

In this paper, a general method, based on DT transformation, is presented to obtain approximate solutions of the linear and the nonlinear differential equations related to engineering problems. This method is called generalized classical method (GCM). The main advantage of the GCM is that it allows to write the solution as an unknown functional series which is suitable for the physical system without having to solve the main differential equation of the system itself. Meanwhile, the unknown coefficients in the series are determined by differential spectrums of the basic differential equation of the system by considering initial and boundary conditions. In the present work, first the essentials and some properties of the DT method and GCM are given and also the application of the GCM is described step by step. Then, as an example, the application of the GCM method to the solution of the nonlinear differential equations which arise in the transient regimes in the electromechanics processes is presented.

Numerical accuracy of the GCM is verified by considering some nonlinear differential equations for which analytical solutions are known. It is observed that the numerical results are in good agreement with the analytical solutions.

\section{Differential transformation (DT) and generalized classical methods (GCM)}

It is well known that linear differential equations with constant coefficients can be transformed into algebraic equations and then easily solved in the complex frequency domain by Laplace or Fourier transformations. For time-varying systems, although the application of these techniques is possible by some modifications, it is not as easy and simple as the former case. For nonlinear differential systems, the problem gets more complex due 
to the frequency-domain convolution integrals that result from the time-domain products of dependent variables or their derivatives. Thus, the use of the standard transform techniques is not practical for nonlinear systems. Fortunately, the use of the DT transform overrides most of the mentioned difficulties and convolution integrals are replaced by simple sums of algebraic terms $[20,21,22,23,24,25,26,27,28]$.

The DT method converts the differential form mathematical model of a system into its spectral form on which algebraic operations can be carried out to derive and understand the system performance. For an analytic function $x(t)$ described by Taylor series

$$
x(t)=\left.\sum_{k=0}^{\infty} \frac{1}{k !} \frac{d^{k} x(t)}{d t^{k}}\right|_{t=0} t^{k},
$$

in the interval $t \in[0, H]$, the spectral model (or transform) is defined to be the discrete function

$$
X(k)=\left.\frac{H^{k}}{k !} \frac{d^{k} x(t)}{d t^{k}}\right|_{t=0},
$$

which is known to be the DT [20, 21, 22, 23, 24, 25]. Using this transform, the Taylor series can be written as

$$
x(t)=\sum_{k=0}^{\infty} X(k)\left(\frac{t}{H}\right)^{k} .
$$

As seen, $X(k)$, the spectrum of $x(t)$, can be obtained by differential transformation and the inverse transform can be obtained by the Taylor series. Therefore, this transformation method is called differential Taylor transformation (DTT) by Pukhov [20]. In general, when the differential spectrums $X(k)$ are known, $x(t)$ can be defined not just as a Taylor series but as a functional series. The functional series can be taken as exponential, Fourier exponential, or any approximation function. Therefore, in general, this method can be defined as differential transformation (DT) method as well. Such an approach expands the applicability of the DT method, and hence makes it possible to get new techniques for solving various engineering problems. One of these techniques, based on the DT method, is generalized classical method (GCM). GCM allows to obtain the solution of the linear and nonlinear differential equations in various types which is similar to the standard method for the solution of the differential equations with constant coefficients. The details of the GCM can be explained as follows.

If the mathematical model is determined as

$$
\frac{d x(t)}{d t}=\varphi(t, x(t)), \quad x(0)=x_{0}
$$

then the approximate analytical solution of this differential equation can be obtained by DT $[25,26,27]$, where $x(t)$ is the basic function vector, $x_{0}$ is the initial value of the function $x(t)$ at $t=0, t$ is the time, $\varphi(t, x(t))$ is the vector function having sufficient number of the derivatives with respect to $x$ and $t$. In general, this function can be nonlinear. 
Although it is nonlinear, according to the basic definition of the GCM if (2.4) has a unique solution, it can be defined as the addition of two components

$$
x(t)=x_{s}(t)+x_{T}(t)
$$

where $x_{s}(t)$ is the main component and $x_{T}(t)$ is the auxiliary component.

Generally, it is possible to choose the component $x_{s}(t)$ as an approximation of any particular solution $(\tilde{x}(t))$ of the system (i.e., $\left.x_{s}(t) \approx \tilde{x}(t)\right)$. For example, $x_{s}(t)$ can be chosen as a value of the system in the steady-state situation $(t \rightarrow \infty)$. This component is independent of the initial situation $(t=0)$ of the system.

The analytical structure of the component $x_{T}(t)$ must be chosen such that the approximation function $x_{T}(t, c) \approx x_{T}(t)$ defining this component becomes convergent. Here, $c=\left(c_{0}, c_{1}, \ldots, c_{n}\right)$ are the undetermined coefficients. These coefficients can be determined from the initial, boundary, or other particular conditions of the considered system. The differential spectrums of (2.4) and (2.5) are defined as follows [20, 21, 22, 23, 24, 25]:

$$
\begin{gathered}
\frac{k+1}{H} X(k+1)=\Phi(k), \quad k=0,1,2, \ldots, \\
X(k)=X_{s}(k)+X_{T}(k),
\end{gathered}
$$

where the differential Taylor spectrums of the main functions $x(t), x_{s}(t), x_{T}(t)$, and $\varphi(t, x(t))$ have the form

$$
\begin{gathered}
X(k)=\left.\frac{H^{k}}{k !} \frac{d^{k} x(t)}{d t^{k}}\right|_{t=0}, \\
X_{s}(k)=\left.\frac{H^{k}}{k !} \frac{d^{k} x_{s}(t)}{d t^{k}}\right|_{t=0}, \\
X_{T}(k)=\left.\frac{H^{k}}{k !} \frac{d^{k} x_{T}(t)}{d t^{k}}\right|_{t=0}, \\
\Phi(k)=\left.\frac{H^{k}}{k !} \frac{d^{k} \varphi(t, x(t))}{d t^{k}}\right|_{t=0},
\end{gathered}
$$

where the initial spectrum (for $k=0)$ is known and $\Phi(0)=\varphi\left(0, x_{0}\right)$.

After choosing the function $x_{s}(t)$ and considering (2.6) and (2.7), the spectrums $X(k)$ and $X_{s}(k)$ can be defined easily using the DT method. Therefore, the spectrums $X_{T}(k)$ in (2.7) can be obtained.

When the function $x_{T}(t)$ is written in the form a suitable approximate function $x_{T}(t, c)$ then

$$
x_{T}(t) \approx x_{T}(t, c)=x_{T}\left(t, c_{0}, b_{0}(t), c_{1}, b_{1}(t), \ldots, c_{n}, b_{n}(t)\right),
$$

where $b_{0}(t), b_{1}(t), \ldots, b_{n}(t)$ are the basis functions which are chosen for the approximate function for the function $x_{T}(t)$. Hence, according to (2.5) and (2.7), the spectrum equation of the function $x(t)$ becomes

$$
X(k)=X_{s}(k)+X_{T}\left(T(k), c_{0}, B_{0}(k), c_{1}, B_{1}(k), \ldots, c_{n}, B_{n}(k)\right),
$$


where $B_{0}(k), B_{1}(k), \ldots, B_{n}(k)$ are the appropriate differential spectrums of the functions $b_{0}(t), b_{1}(t), \ldots, b_{n}(t)$.

The spectrums $T(k)$ can be determined as follows:

$$
T(k)=\left.\frac{H^{k}}{k !} \frac{d^{k} t}{d t^{k}}\right|_{t=0}=H \delta(k-1)=(0, H, 0, \ldots, 0),
$$

where

$$
\delta(k)= \begin{cases}1 & \text { if } k=0 \\ 0 & \text { if } k \neq 0\end{cases}
$$

or

$$
\delta(k-n)= \begin{cases}1 & \text { if } k=n \\ 0 & \text { if } k \neq n\end{cases}
$$

When considering initial and boundary conditions of the system in (2.10), the coefficients $c_{0}, c_{1}, \ldots, c_{n}$ can be defined with respect to the spectrums $X(k)$ and $X_{s}(k)$. However, in this case from (2.5), it can be said that the approximate analytical solution of the system is obtained. Therefore, in the GCM, using DT, the approximate analytical solution of (2.4) can be obtained in form (2.5) without having to find its analytical solution.

Consequently, the investigation of the transient regimes in the physical systems can be implemented by the GCM according to the following procedure [26, 27, 28].

(1) The mathematical model of the considered system is formed by using the physical laws. This model consists of linear or nonlinear differential equations.

(2) The spectrum models of these differential equations can be obtained using the DT method $[20,21,22,23,24,25,26,27,28]$. During this process, the original functions are included in these equations' transform to the differential spectrums.

(3) The spectrums $X(k)$ are obtained from the spectrum models of the differential equations, that is, $X(0)=x_{0}, X(1), \ldots, X(n)$, where $n$ is the number of the spectrums which are used in the calculations.

(4) The main component $x_{s}(t)$ is determined and its DT spectrums $X_{s}(k)$ are calculated. Hence, the differential spectrums of the auxiliary component can be determined in form $X_{T}(k)=X(k)-X_{s}(k)$, which is appropriate in (2.7).

(5) According to the components $X_{T}(k)$, the original of the auxiliary component $x_{T}(t)$ is determined. If the auxiliary component $x_{T}(t)$ is chosen as an approximate function, that is,

$$
x_{T}(t) \approx x_{T}\left(t, c_{0}, c_{1}, c_{2}, \ldots, c_{n}\right),
$$

then the undetermined coefficients $c_{0}, c_{1}, \ldots, c_{n}$ of this function can be determined from the following equilibrium equation:

$$
X(k)-X_{s}(k)=X_{T}(k)=X_{T}\left(T(k), c_{0} B_{0}(k), c_{1} B_{1}(k), \ldots, c_{n} B_{n}(k)\right) .
$$


The coefficients $c_{0}, c_{1}, \ldots, c_{n}$ can be calculated easily according to the certain spectrums $X_{T}(k)$ for $k=0,1,2, \ldots, n$. Hence, the component $x_{T}(t)$ can be expressed as

$$
x_{T}(t) \approx x_{T}\left(t, c_{1}, c_{2}, \ldots, c_{n}\right)=c_{0} b_{0}(t)+c_{1} b_{1}(t)+\cdots+c_{n} b_{n}(t) .
$$

(6) The solution of the differential equation of the considered system can be obtained by adding the originals of the main and auxiliary components: $x(t)=$ $x_{s}(t)+x_{T}(t, c)$.

When the above procedure is analyzed, it can be seen that it is possible to obtain the analytical or approximate analytical solution of the various linear and the nonlinear differential equations using the GCM. In this case, it is not necessary to solve the main equation, we just need to calculate the DT spectrums of the original equation. On the other hand, the effect of the GCM depends on choosing appropriate components $x_{s}(t)$ and $x_{T}(t)$. In general, in the solution of any differential equation, it may be difficult to choose $x_{s}(t)$ and $x_{T}(t)$ directly. However, the GCM can be used effectively for investigation of the transient or nonstationary regimes occurring in the physical processes. Because the properties of the processes in the physical systems (periodic, nonperiodic, exponential, etc.) are known, $x_{s}(t)$ and $x_{T}(t)$ can be expressed easily using these properties.

\section{The investigation of the transient regimes by the GCM}

The GCM can be used effectively for the analysis of the transient regimes occurring in the physical processes (such as mechanics, electromechanics, heat-mass transfer, and fluid dynamics, etc.). In this section, three concrete examples are given to investigate transient regimes in the electromechanics processes, using the GCM.

Example 3.1. We present an analysis of the transient events on nonlinear resistance inductance (RL) electrical circuit connected to the direct current (DC) supply by the GCM.

If the effect of the eddy-current losses is ignored, then the transient event in the mentioned circuit RL can be written in terms of dimensionless parameters as follows $[18,25]$ :

$$
\frac{d x}{d \tau}+x^{m}=u
$$

where $x$ is the magnetic flux ratio, $u$ is the applied voltage ratio (is constant), $\tau$ is the dimensionless time.

In general, (3.1) is nonlinear, but it has an analytic solution for any value of the integer $m$. The cases $m=1$ and 3 are important for the electromechanics systems [18]. Therefore, we will consider the solutions in these cases. For the validation of the obtained results, we consider the analytical solution of (3.1) for $m=1$ and 3 with zero initial condition.

For $m=1$,

$$
\tau=\ln \frac{u}{u-x}
$$


For $m=3$,

$$
\tau=\frac{1}{6 u^{2 / 3}} \ln \left[\frac{u^{2 / 3}+u^{1 / 3} x+x^{2}}{\left(u^{1 / 3}-x\right)^{2}}\right]+\frac{1}{u^{2 / 3} \sqrt{3}}\left(\tan ^{-1} \frac{u^{1 / 3}+2 x}{u^{1 / 3} \sqrt{3}}-\tan ^{-1} \frac{1}{\sqrt{3}}\right) .
$$

Now, we investigate the GCM solution of (3.1) in the circuit RL. The steady-state value of the current or flux according to $(3.1)$ is $(t \rightarrow \infty)$,

$$
x_{s}(\tau)=\sqrt[m]{u}
$$

To determine the auxiliary component $x_{T}(\tau)$, we need the DT of $(3.1)$ :

$$
\frac{k+1}{H} X(k+1)+X^{(m)}(k)=u \delta(k)
$$

where

$$
X^{(m)}(k)=\sum_{l=0}^{k} X(k-l) X^{(m-1)}(l) .
$$

For the auxiliary component $x_{T}(\tau)$, approximate functional series can be chosen as (because $x(\tau) \rightarrow 0$ as $\tau \rightarrow \infty$ )

$$
x_{T}(\tau)=-\sqrt[m]{u}\left(\frac{1}{1+c_{1} \tau+c_{2} \tau^{2}+\cdots+c_{n} \tau^{n}}\right) .
$$

Considering (2.5), (3.4), and (3.7), the approximate solution of (3.1) is

$$
x(\tau)=\sqrt[m]{u}\left(\frac{c_{1} \tau+c_{2} \tau^{2}+\cdots+c_{n} \tau^{n}}{1+c_{1} \tau+c_{2} \tau^{2}+\cdots+c_{n} \tau^{n}}\right) .
$$

The coefficients $c_{1}, c_{2}, \ldots, c_{n}$ can be obtained from (3.5) and (3.8). For this purpose, (3.8) can be written as

$$
x(\tau)\left(1+c_{1} \tau+c_{2} \tau^{2}+\cdots+c_{n} \tau^{n}\right)=\sqrt[m]{u}\left(c_{1} \tau+c_{2} \tau^{2}+\cdots+c_{n} \tau^{n}\right) .
$$

From (3.5) and (3.9), the DT spectrum model of the system becomes as follows:

$$
\begin{gathered}
\frac{k+1}{H} X(k+1)+X^{(m)}(k)=u \delta(k), \\
X(k)+c_{1} H X(k-1)+\cdots+c_{n} H^{n} X(k-n) \\
=\sqrt[m]{u}\left(c_{1} H \delta(k-1)+c_{2} H^{2} \delta(k-2)+\cdots+c_{n} H^{n} \delta(k-n)\right) .
\end{gathered}
$$

Since $X(0)=0$, the coefficients $c_{1}, c_{2}, \ldots, c_{n}$ can be denoted for $k=0,1,2, \ldots, n$. Hence, we obtain the approximate solution of (3.1) from (3.8). This procedure is as follows for the cases $m=1$ and $m=3$.

For $m=1$,

$$
\begin{gathered}
X(0)=0, \quad X(1)=u H, \quad X(2)=-\frac{u H^{2}}{2 !}, \quad X(3)=\frac{u H^{3}}{3 !}, \ldots, \\
c_{1}=1, \quad c_{2}=\frac{1}{2 !}, \quad c_{3}=\frac{1}{3 !}, \quad c_{4}=\frac{1}{4 !}, \ldots
\end{gathered}
$$




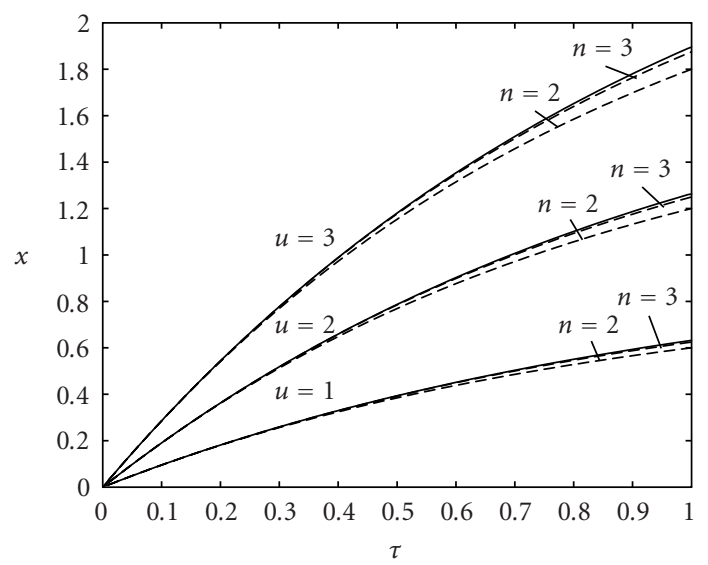

Figure 3.1. The variations of the magnetic flux in transient regime at various terminal voltages for $m=1$. Solid lines: analytical solutions, dashed lines: GCM solutions (Example 3.1).

From (3.8), we obtain

$$
x(\tau)=u\left(1-\frac{1}{1+\tau+\tau^{2} / 2 !+\tau^{3} / 3 !+\cdots}\right) .
$$

For $m=3$, the exact expression of $X^{(m)}(k)$ in (3.10) is

$$
X^{(3)}(k)=\sum_{l=0}^{k} X(k-l) \sum_{s=0}^{l} X(l-s) X(s) \text {. }
$$

Considering (3.14), from (3.10) and (3.11), we can denote the differential spectrum $X(k)$ and the coefficients $c_{1}, c_{2}, \ldots, c_{n}$ for the case $m=3$ :

$$
\begin{gathered}
X(0)=0, \quad X(1)=u H, \quad X(2)=0, \quad X(3)=0, \quad X(4)=-\frac{u^{3} H^{4}}{4}, \ldots, \\
c_{1}=u^{2 / 3}, \quad c_{2}=u^{4 / 3}, \quad c_{3}=u^{6 / 3}, \quad c_{4}=\frac{3}{4} u^{8 / 3}, \ldots
\end{gathered}
$$

From (3.8) and (3.15), we obtain

$$
x(\tau)=u^{1 / 3}\left(1-\frac{1}{1+u^{2 / 3} \tau+u^{4 / 3} \tau^{2}+u^{6 / 3} \tau^{3}+(3 / 4) u^{8 / 3} \tau^{4}+\cdots}\right) .
$$

For $u=1,2,3$, the comparison of the results that are obtained from (3.2), (3.3), (3.13), and (3.16) is shown in Figures 3.1 and 3.2 for $m=1$ and $m=3$, respectively. As can be seen the analytical and the approximate solutions obtained by the GCM are in good agreement. However, it is clear that (3.13) and (3.16) are more simple than (3.2) and (3.3) from the point of view of engineering analysis. In particular, it can be observed that the analysis of the transient regimes of the system becomes difficult using (3.2) and (3.3) as $x \rightarrow u^{1 / m}$. 


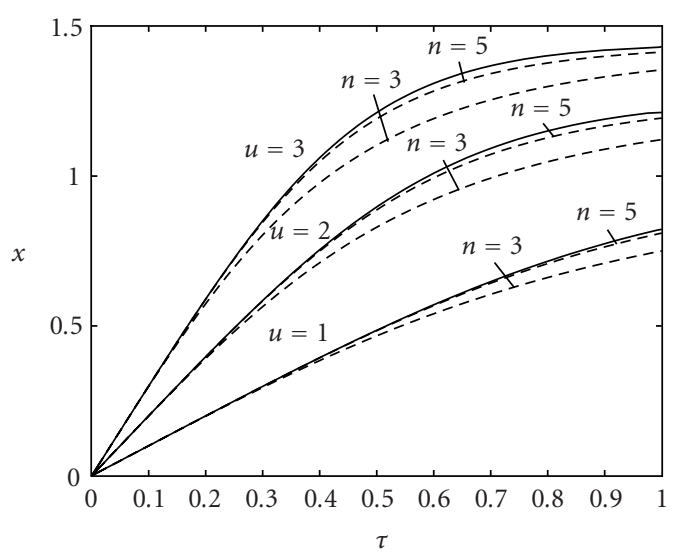

Figure 3.2. The variations of the magnetic flux in transient regime at various voltages for $m=3$. Solid lines: analytical solutions, dashed lines: GCM solutions (Example 3.1).

Furthermore, in the solution of the problem, we can choose the function $x_{T}(\tau)$ not only as an inverse of a power series but also as any function. For example, function $x_{T}(\tau)$ can be chosen as an exponential power series function, that is,

$$
x_{T}(\tau)=e^{-\alpha \tau}\left(c_{0}+c_{1} \tau+c_{2} \tau^{2}+\cdots\right),
$$

where coefficients $\alpha, c_{0}, c_{1}, \ldots$ must be determined.

Example 3.2. We present an investigation of the variation of magnetic flux or electromotor force of the DC motor driver operating as a generator.

Ignoring the effect of the eddy-current losses, the differential equation of this process in dimensionless parameters can be written as [18]

$$
\frac{d x}{d \tau}+y(x)=u
$$

where $x$ is the magnetic flux ratio, $y$ is the current ratio (nonlinear dependence on flux ratio), $u$ is the applied voltage ratio, $\tau$ is the dimensionless time.

The magnetization curves of the driver machines can be defined by

$$
x=\frac{y}{a y+b},
$$

where $a$ and $b$ are constants [18].

Considering (3.19), we can write (3.18) as follows:

$$
(1-a x) \frac{d x}{d \tau}+\lambda x=u
$$

where $\lambda=a u+b$. 
512 The investigation of the transient regimes

Its analytical solution with the initial condition $x(0)=0$ is

$$
\tau=\frac{1}{\lambda^{2}}\left[b\left(\ln \frac{u}{u-\lambda x}\right)+a \lambda x\right]
$$

Although it looks simple, (3.21) has not enough advantage in the analytical investigation of $x=f(\tau)$. This difficulty can be solved using the GCM.

We suppose that the stationary solution of (3.20) is $x_{s}(\tau)=u / \lambda$ and its auxiliary component is as in (3.7):

$$
x_{T}(\tau)=-\frac{u}{\lambda}\left(\frac{1}{1+c_{1} \tau+c_{2} \tau^{2}+\cdots+c_{n} \tau^{n}}\right) .
$$

Thus the approximate analytical solution of (3.20) is

$$
x(\tau)=\frac{u}{\lambda}\left(\frac{c_{0} \tau+c_{2} \tau^{2}+\cdots+c_{n} \tau^{n}}{1+c_{1} \tau+c_{2} \tau^{2}+\cdots+c_{n} \tau^{n}}\right) .
$$

Thus the DT spectrum model of the system which is formed by (3.20) and (3.23) becomes as follows:

$$
\begin{gathered}
\frac{k+1}{H} X(k+1)-\frac{a}{H} \sum_{l=0}^{k}(l+1) X(l+1) X(k-l)+\lambda X(k)=u \delta(k), \\
X(k)+c_{1} H X(k-1)+\cdots+c_{n} H^{n} X(k-n) \\
=\frac{u}{\lambda}\left[c_{1} H \delta(k-1)+\cdots+c_{n} H^{n} \delta(k-n)\right] .
\end{gathered}
$$

The spectrums $X(k)$ and coefficients $c_{n}$ can be determined by taking $k=0,1,2, \ldots, n$ :

$$
\begin{gathered}
X(0)=0, \quad X(1)=u H, \quad X(2)=-\frac{u b}{2 !} H^{2}, \quad X(3)=\frac{u b}{3 !}(b-2 a u) H^{3}, \ldots, \\
c_{1}=\lambda, \quad c_{2}=\lambda\left(\lambda-\frac{b}{2}\right), \quad c_{3}=\lambda^{3}-\lambda^{2} b+\frac{\lambda b}{6}(b-2 a u), \ldots .
\end{gathered}
$$

Figures 3.3, 3.4, and 3.5 show the variations of the magnetic flux in the field coil of the machine at various terminal voltages $u=1,2,3$ and temporary regimes for the values $a=$ 0.728 and $b=0.28$ [18]. As can be seen in the large interval of time, there is a reasonable agreement between (3.21) and its approximate solution (3.23).

In practical view, the investigation of the transfer functions is very important in the control of the electrical driver machine. For the determination of the transfer function, it is necessary to solve (3.18) and (3.19) with respect to the current ratio $y$. Therefore, after some simple calculations, the equation of the driver machine becomes

$$
b \frac{d y}{d \tau}+(a y+b)^{2}(y-u)=0, \quad y(0)=0 .
$$




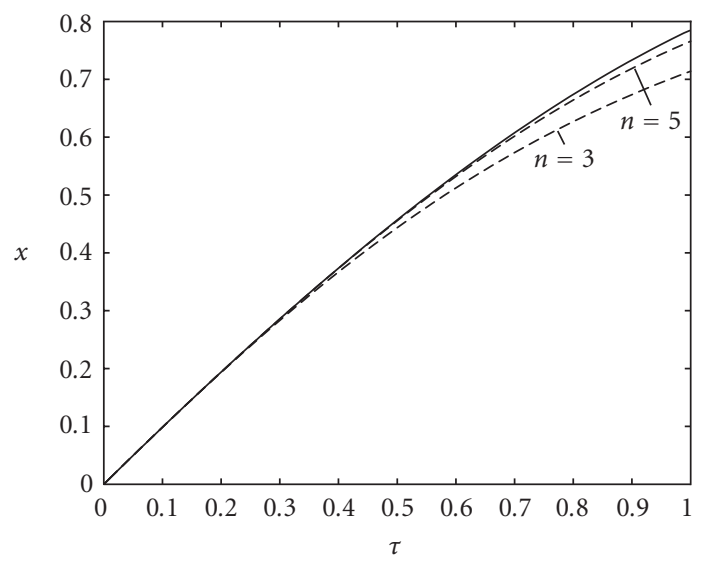

Figure 3.3. The variations of the magnetic flux in the field at the machine for the terminal voltage $u=1$. Solid line: analytical solutions, dashed lines: GCM solutions (Example 3.2).

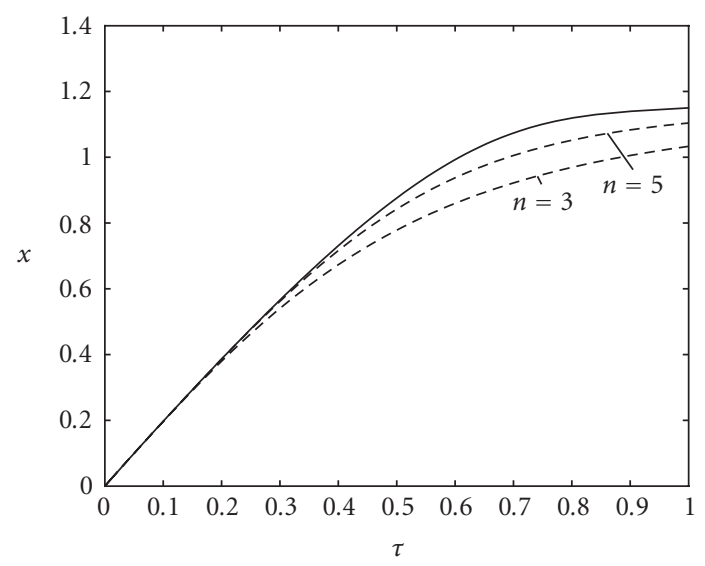

Figure 3.4. The variations of the magnetic flux in the field at the machine for the terminal voltage $u=2$. Solid line: analytical solutions, dashed lines: GCM solutions (Example 3.2).

Equation (3.26) is a nonlinear equation and it has an analytical solution:

$$
\tau=\frac{1}{\lambda}\left(\frac{a y}{a y+b}\right)+\frac{b}{\lambda^{2}} \ln \left(\frac{u}{u-y}\right)+\frac{b}{\lambda^{2}} \ln \left(\frac{a y+b}{b}\right) .
$$

However, this analytical solution is a complicated expression so it is not useful for the engineering analysis of the transient regimes. Therefore, we can obtain more simple approximate solution of (3.26) by the GCM. It is also possible to obtain DT approximate solutions of (3.26) according to the above procedure. But, to show the possibilities of the GCM, we represent the auxiliary function $y_{T}(\tau)$ by another functional series, for example, the exponential power series as in (3.17). In this case, $y_{T}(\infty)=0$, and hence 
514 The investigation of the transient regimes

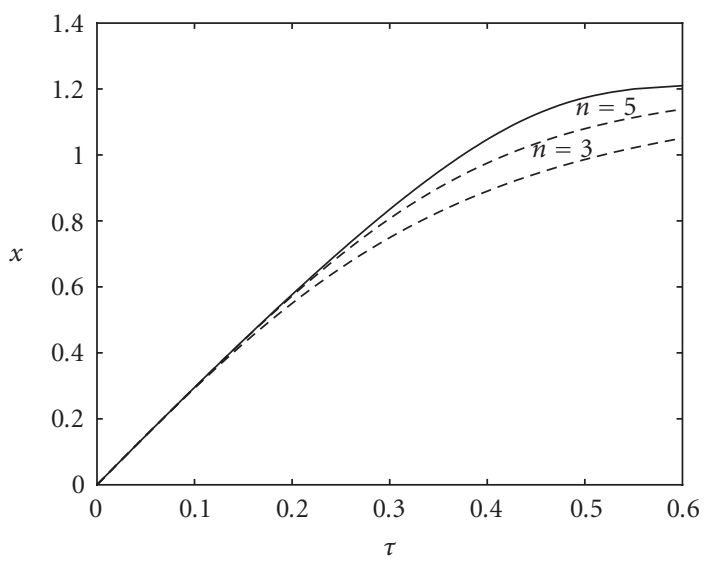

Figure 3.5. The variations of the magnetic flux in the field at the machine for the terminal voltage $u=3$. Solid line: analytical solutions, dashed lines: GCM solutions (Example 3.2).

$y_{T}(\tau)$ can be written as

$$
y_{T}(\tau)=e^{-\alpha \tau}\left(c_{0}+c_{1} \tau+c_{2} \tau^{2}+\cdots+c_{n} \tau^{n}\right),
$$

where $\alpha, c_{0}, c_{1}, c_{2}, \ldots, c_{n}$ are the unknown coefficients.

As follows from (3.26) at $\tau=\infty, y_{s}(\tau)=u$. Thus, the approximate solution of (3.26) is

$$
y(\tau)=y_{s}(\tau)+y_{T}(\tau)=u+e^{-\alpha \tau}\left(c_{0}+c_{1} \tau+c_{2} \tau^{2}+\cdots+c_{n} \tau^{n}\right) .
$$

Before the coefficients $\alpha, c_{1}, c_{2}, \ldots, c_{n}$ are determined by DT spectrums, we note that the following relations can be derived from (3.26) and (3.29):

$$
c_{0}=-u, \quad c_{1}=u(b-\alpha),\left.\quad \frac{d y}{d \tau}\right|_{\tau=0}=b u .
$$

We write the (3.26) and (3.29) in the form

$$
\begin{gathered}
\frac{d y}{d \tau}+\frac{a^{2}}{b} y^{3}+\left(2 a-\frac{a^{2} u}{b}\right) y^{2}+(b-2 a u) y=b u, \\
(y-u) e^{\alpha \tau}=c_{0}+c_{1} \tau+c_{2} \tau^{2}+\cdots+c_{n} \tau^{n} .
\end{gathered}
$$

The DT spectrums can be determined from (3.31):

$$
\begin{aligned}
& \frac{k+1}{H} Y(k+1)+\frac{a^{2}}{b} \sum_{l=0}^{k} Y(k-l) \sum_{s=0}^{l} Y(l-s) Y(s) \\
& \quad+\left(2 a-\frac{a^{2} u}{b}\right) \sum_{l=0}^{k} Y(k-l) Y(l)+(b-2 a u) Y(k)=b u \delta(k), \\
& \sum_{l=0}^{k} \frac{(\alpha H)^{k-l}}{(k-l) !} Y(l)-u \frac{(\alpha H)^{k}}{k !}=c_{0} \delta(k)+c_{1} H \delta(k-1)+\cdots
\end{aligned}
$$




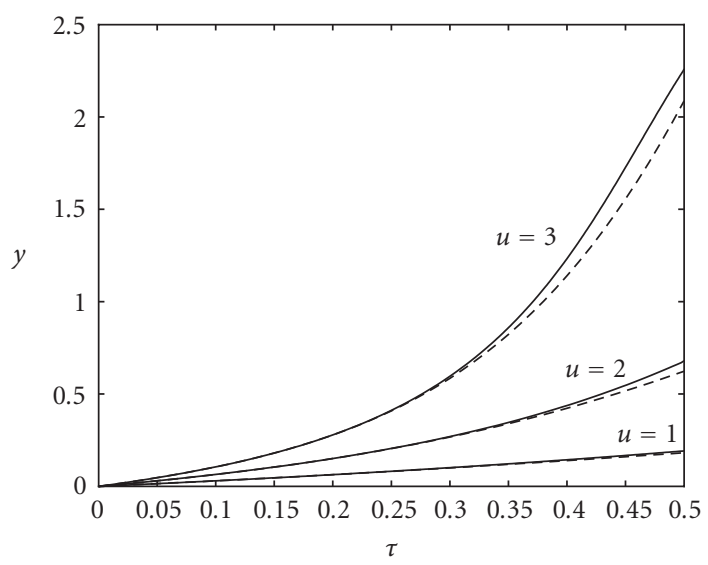

Figure 3.6. The variations of the magnetic field current of the machine at various terminal voltages. We have taken $n=3,6$, and 8 for $u=1,2$, and 3, respectively. Solid lines: analytical solutions, dashed lines: GCM solutions (Example 3.2).

So the coefficients $c_{1}, c_{2}, \ldots, c_{n}$ in (3.29) can be determined by (3.32) and (3.33) as follows:

$$
\begin{gathered}
c_{m}=\frac{1}{H^{m}}\left[\sum_{l=0}^{m} \frac{(\alpha H)^{m-l}}{(m-l) !} Y(l)-u \frac{(\alpha H)^{m}}{m !}\right], \quad m=0,1,2, \ldots, n, \\
Y(0)=0, \quad Y(1)=b u H,
\end{gathered}
$$

where the differential spectrums $Y(2), Y(3), \ldots$ can be determined from (3.32). Thus, after some straightforward calculations, the coefficients $c_{1}, c_{2}, \ldots, c_{n}$ can be obtained from (3.32) and (3.34):

$$
\begin{gathered}
c_{0}=-u, \quad c_{1}=u(b-\alpha), \quad c_{2}=\frac{b u}{2 !}\left(2 \alpha-b+2 a u-\frac{\alpha^{2}}{b}\right), \\
c_{3}=\frac{b u}{3 !}\left[3 \alpha^{2}+(b-4 a u)^{2}-3 \alpha(b-2 a u)-10(a u)^{2}-\frac{\alpha^{3} u}{b}\right] .
\end{gathered}
$$

In general, the parameter $\alpha>0$ can be chosen according to the convergence condition of the functional series or arbitrarily. For example, it can be taken as $\alpha=2 \lambda$.

A comparison of the analytical solution in (3.27) and the approximate solution of the problem (3.29) are shown in Figure 3.6 for the values $u=1,2,3, a=0.728$, and $b=0.28$. The results are in good agreement, however, it is clear that (3.29) is simple and has an advantage for the theoretical and practical investigations.

It can be very difficult to investigate the transient regimes of the electrodynamics processes in the electrical machine driver. However, in the following example, it is shown that GCM allows to study the transient events in such regimes.

Example 3.3. We present an analysis of the skidding of the wheel pair in an electrical train with a separate excitation DC machine which has a rheostat characteristic. 
Suppose that the instantaneous change of the terminal voltage is caused by the skidding and at this moment, the velocity of the electrical train does not change. Therefore, the equation of the transient regime of the electrodynamics of the electrical train becomes [18]

$$
j(1+a x) \frac{d x}{d \tau}+a x^{2}+b x+c=0,
$$

where $a, b, c$, and $j$ are the coefficients which are dependent on the electrical, mechanical, and geometrical parameters of the system, $x$ is the relatively slip at the skidding, $\tau$ is the dimensionless time.

Equation (3.36) is also a nonlinear differential equation. Its analytical solution corresponding to zero initial condition is

$$
\tau=\frac{j}{2} \ln \left(\frac{c}{a x^{2}+b x+c}\right)+\frac{j}{2}\left[\frac{2-b}{a\left(x_{1}-x_{2}\right)}\right]+\ln \left[\frac{\left(x-x_{2}\right) x_{1}}{\left(x-x_{1}\right) x_{2}}\right],
$$

where $x_{1}$ and $x_{2}$ are the real roots of the equation

$$
a x^{2}+b x+c=0
$$

We obtain the approximate solution of (3.36) by using the GCM. To do this, we choose the approximate auxiliary function as in (3.28). Thus, the DT spectrum model is formed as follows:

$$
x(\tau)=x_{s}(\tau)+x_{T}(\tau)=x_{s}(\tau)+e^{-\alpha \tau}\left(c_{0}+c_{1} \tau+c_{2} \tau^{2}+\cdots\right) .
$$

We suppose that the component of the slip is

$$
x_{s}(\tau=\infty)=x_{1}
$$

where $x_{1}>0$ is a root of (3.38). At the zero initial condition,

$$
x(0)=0, \quad x_{s}(0)+c_{0}=0,\left.\quad \frac{d x}{d \tau}\right|_{\tau=0}=-\alpha c_{0}+c_{1}=\frac{-c_{1}}{j} .
$$

Now we can form the DT spectrum model of the problem from (3.36) and (3.39):

$$
\begin{aligned}
\frac{k+1}{H} X(k+1)+\frac{a}{H} \sum_{l=0}^{k}(l+1) X(l+1) X(k-l)+\frac{a}{j} \sum_{l=0}^{k} X(k-l) X(l) \\
\quad+\frac{b}{j} X(k)+\frac{c}{j} \delta(k)=0, \\
c_{m}=\frac{1}{H^{m}}\left[\sum_{l=0}^{m} \frac{(\alpha H)^{m-l}}{(m-l) !} X(l)-x_{1} \frac{(\alpha H)^{m}}{m !}\right], \quad m=0,1,2, \ldots, n .
\end{aligned}
$$


If we consider the differential spectrums in (3.42), then the unknown coefficients $c_{0}, c_{1}, c_{2}, \ldots, c_{n}$ can be determined from (3.43):

$$
\begin{gathered}
X(0)=0, \quad X(1)=-\frac{c H}{j}, \quad X(2)=-\frac{c H^{2}}{2 ! j^{2}}(a c-b), \\
X(3)=-\frac{c H^{3}}{3 ! j^{3}}\left[(2 a c-b)^{2}-(a c)^{2}+2 a c\right], \ldots, \\
c_{0}=-x_{1}, \quad c_{1}=-\frac{c}{j}-\alpha x_{1}, \quad c_{2}=-\frac{\alpha c}{j}-\frac{c}{2 j^{2}}(a c-b)-\frac{\alpha^{2}}{2} x_{1}, \ldots
\end{gathered}
$$

Since the coefficient $\alpha>0$ in (3.44) designates the number of terms of the power series, it can be chosen arbitrarily or according to the special situation of the system. Therefore, if the number of terms of the power series in (3.39) is kept fixed, then $\alpha$ can be defined easily. For example, if we suppose that $c_{2}=0$, then from (3.44),

$$
\alpha=\frac{-c}{j x_{1}}\left[\sqrt{1-\frac{x_{1}}{c}(a c-b)}+1\right] .
$$

Thus, for the real parameters which are obtained from the practical applications [18]: $j=0.6896, a=15.9322, b=-14.3725, c=-0.1656, x_{1}=0.9138$, and $x_{2}=-0.01137$, we get

$$
\alpha=2.39, \quad c_{0}=-0.9138, \quad c_{1}=-1.9438 .
$$

Therefore, from (3.39), (3.40), and (3.46), the slip on the electrical train becomes

$$
x(\tau)=0.9138-e^{-2.39 \tau}(0.9138+1.9438 \tau) .
$$

The approximate solution that has been obtained is similar to the one given in [18] which is obtained by a more complicated method.

In Figure 3.7, the slip curves which occur during the skidding of the wheel pairs of the electrical train are given according to the GCM (3.47) and the analytical solution (3.37). From Figure 3.7, it can be seen that the results are in good agreement for a large interval of $x$ and $\tau$. However, it is certain that the approximate solution obtained by the GCM is more useful than the analytical solution of the problem in terms of practical calculations.

\section{Conclusions}

In this paper, an application of the GCM based on the DT method to the nonlinear differential equations has been investigated. The advantages and features of the GCM can be summarized as follows.

(1) The DT method can be used to obtain both numerical and analytical solutions of both linear and nonlinear differential equations.

(2) The GCM allows us to obtain the analytical or approximate analytical solutions (as functional series) of the differential equations. In this method, in general, the solutions of both linear and nonlinear differential equations can be obtained 


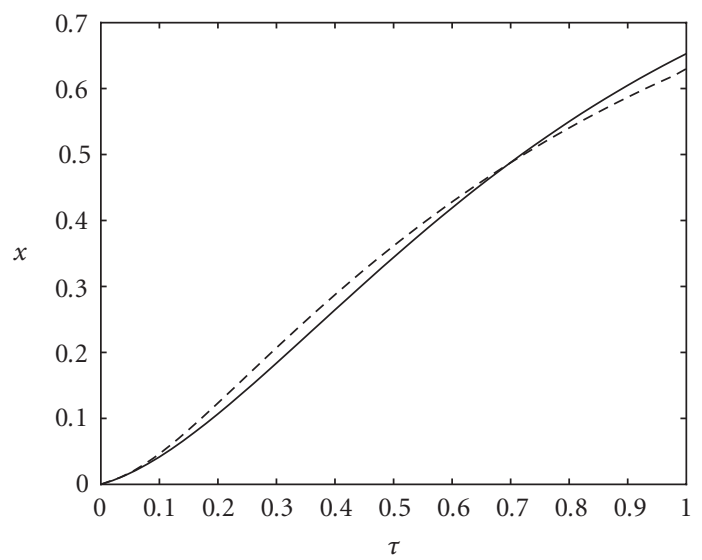

Figure 3.7. Dependence of the slip curves at the skidding of the wheel pairs of an electrical train at starting. Solid line: analytical solution, dashed line: GCM solution (Example 3.3).

using the same solution procedure. The solution of the differential equation consists of two components: main and auxiliary.

(3) The advantage of the GCM is determining the unknown coefficients in the auxiliary component by using the differential spectrums of the variables. In this case, it is not necessary to have the analytical solution of the main differential equation.

(4) When the properties (periodic, nonperiodic, monotonous increasing or decreasing) of the processes in the physical systems are considered, it becomes easy to choose the functional structures of the main and auxiliary components. Therefore, it is expected that the GCM will be effective in the modeling of the transient regimes in the linear and nonlinear physical systems.

(5) Some papers that appeared in the literature show that the DT method can be used to solve two- and three-dimensional differential equations. Therefore, the GCM can be extended to solve these equations.

We conclude that the GCM combined with DT is eminently suitable for the numerical solutions of the ordinary and partial differential equations that arise from several science areas.

\section{References}

[1] T. Abbasov, S. Herdem, and M. Köksal, Modelling of distributed parameter nonlinear systems by differential Taylor method, Control Cybernet. 28 (1999), no. 2, 259-267.

[2] I. H. Abdel-Halim Hassan, Different applications for the differential transformation in the differential equations, Appl. Math. Comput. 129 (2002), no. 2-3, 183-201.

[3] - On solving some eigenvalue problems by using a differential transformation, Appl. Math. Comput. 127 (2002), no. 1, 1-22.

[4] F. Ayaz, On the two-dimensional differential transform method, Appl. Math. Comput. 143 (2003), no. 2-3, 361-374.

[5] Applications of differential transform method to differential-algebraic equations, Appl. Math. Comput. 152 (2004), no. 3, 649-657. 

, Solutions of the system of differential equations by differential transform method, Appl. Math. Comput. 147 (2004), no. 2, 547-567.

[7] S. C. Chapra and R. P. Canale, Numerical Methods for Engineers, McGraw-Hill, New York, 1990.

[8] C.-K. Chen and S.-H. Ho, Application of differential transformation to eigenvalue problems, Appl. Math. Comput. 79 (1996), no. 2-3, 173-188.

[9] Solving partial differential equations by two-dimensional differential transform method, Appl. Math. Comput. 106 (1999), no. 2-3, 171-179.

[10] , Transverse vibration of a rotating twisted Timoshenko beams under axial loading using differential transform, Mechanical Sciences 41 (1999), 1339-1356.

[11] C.-L. Chen and Y.-C. Liu, Solution of two-point boundary-value problems using the differential transformation method, J. Optim. Theory Appl. 99 (1998), no. 1, 23-35.

[12] S.-H. Ho and C.-K. Chen, Analysis of general elastically and restrained non-uniform beams using differential transform, Appl. Math. Modelling 22 (1998), 219-234.

[13] M.-J. Jang and C.-L. Chen, Analysis of the response of a strongly nonlinear damped system using a differential transformation technique, Appl. Math. Comput. 88 (1997), no. 2-3, 137-151.

[14] M.-J. Jang, C.-L. Chen, and Y.-C. Liu, On solving the initial-value problems using the differential transformation method, Appl. Math. Comput. 115 (2000), no. 2-3, 145-160.

[15] _ Two-dimensional differential transform for partial differential equations, Appl. Math. Comput. 121 (2001), no. 2-3, 261-270.

[16] M.-J. Jang, J.-S. Wang, and Y.-C. Liu, Applying differential transformation method to parameter identification problems, Appl. Math. Comput. 139 (2003), no. 2-3, 491-502.

[17] M. Köksal and S. Herdem, Analysis of nonlinear circuits by using differential Taylor transform, Comput. Electr. Engrg. 28 (2002), 513-525.

[18] S. I. Karibov and P. F. Merabischvili, Approximation analytical solution method of nonlinear differential equations in traction electrical drivers analysis, Electricity 5 (2001), 35-40.

[19] A. H. Nayfeh, Perturbation Methods, Wiley-Interscience [John Wiley \& Sons], New York, 2000.

[20] G. E. Pukhov, Taylor Transformation and Its Applications for Electrotechnics and Electronics, Naukova Dumka, Kiev, 1978.

[21] Differential Transforms Functions and Equations, Naukova Dumka, Kiev, 1980.

[22] _ Differential Analysis of Electrical Circuits, Naukova Dumka, Kiev, 1982.

[23] Differential Transformation and Mathematical Modeling of Physical Processes, Naukova Dumka, Kiev, 1986.

[24] Approximation Methods of the Mathematical Modeling by Differential T-Transform, Naukova Dumka, Kiev, 1988.

[25] _ Differential Spectrums and Models, Naukova Dumka, Kiev, 1990.

[26] Examples of non-stationary process simulation by generalized classical method, Electronnoe Modelirovanie 12 (1990), 90-94 (Russian).

[27] Calculations of transients in the nonlinear electrical circuits with pulse sources, Electronnoe Modelirovanie 13 (1991), 44-50 (Russian).

[28] Mathematical Modeling and analysis of transient regimes in complex physical technical systems by differential transformations, Electronic Modeling 6 (1994), 44-46.

[29] M. Rahman, Mathematical Methods with Applications, WIT Press, Southampton, 2000.

T. Abbasov: Department of Electrical and Electronic Engineering, Faculty of Engineering, İnönü University, 44280 Malatya, Turkey

E-mail address: tabbasov@inonu.edu.tr

A. R. Bahadir: Department of Mathematics, Faculty of Arts and Sciences, İnönü University, 44280 Malatya, Turkey

E-mail address: arbahadir@inonu.edu.tr 


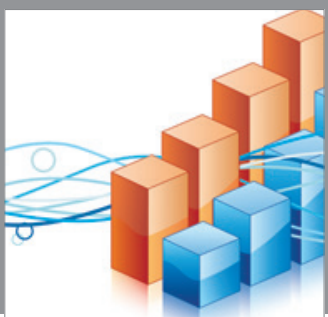

Advances in

Operations Research

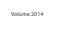

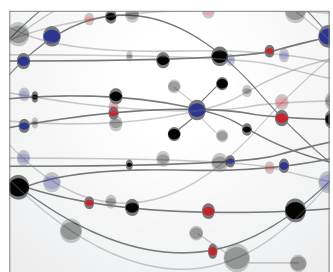

\section{The Scientific} World Journal
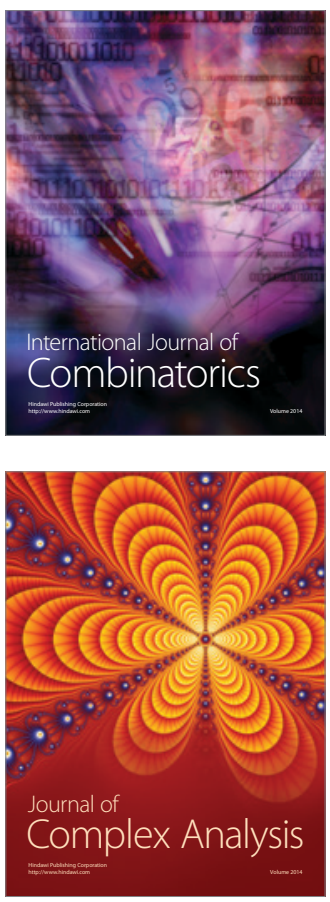

International Journal of

Mathematics and

Mathematical

Sciences
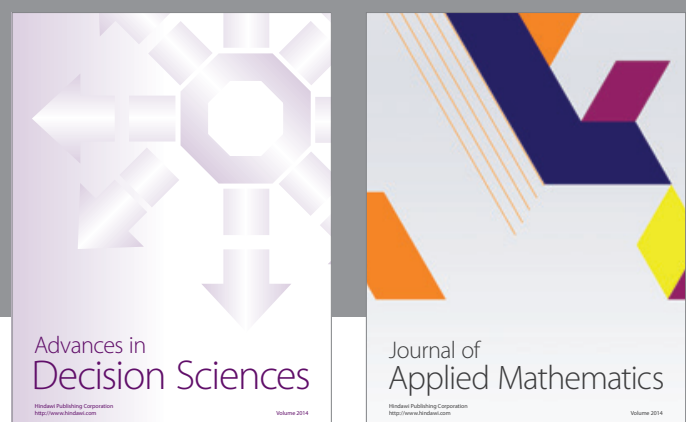

Journal of

Applied Mathematics
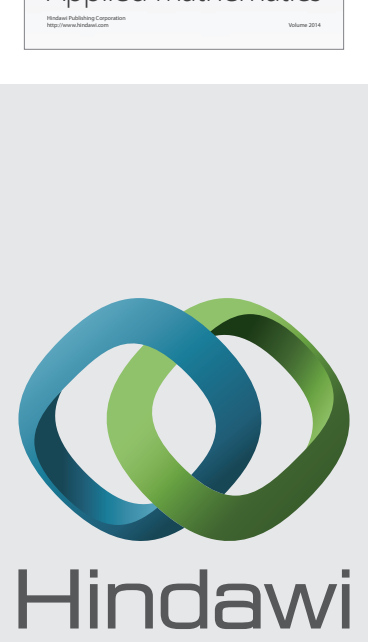

Submit your manuscripts at http://www.hindawi.com
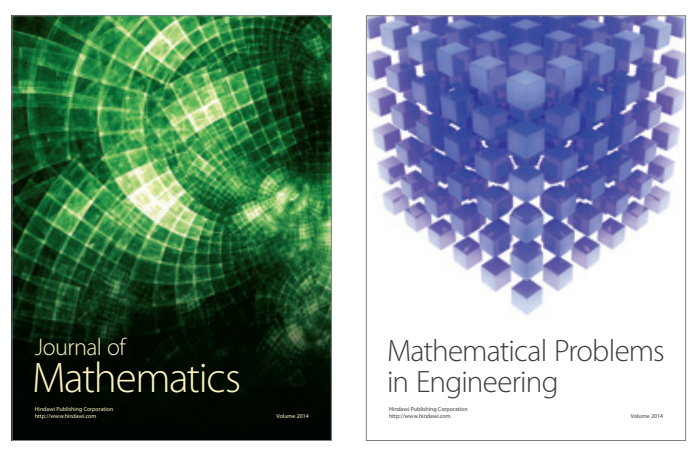

Mathematical Problems in Engineering
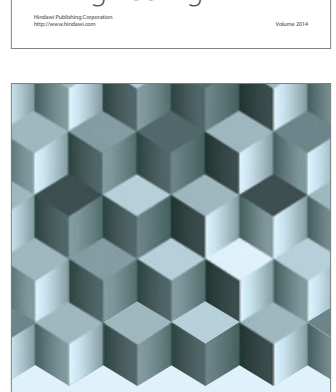

Journal of

Function Spaces
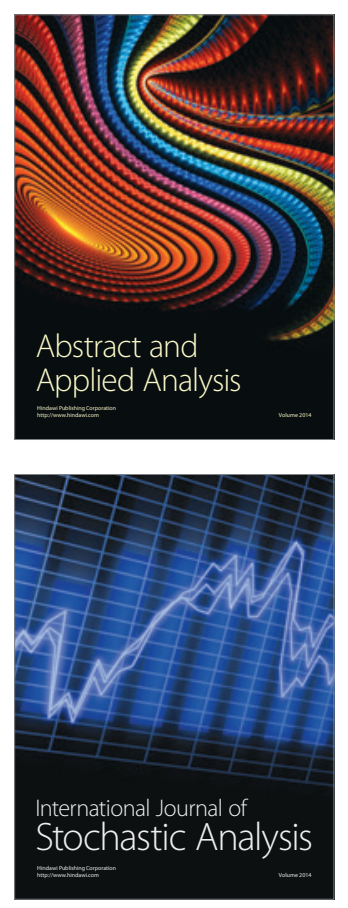

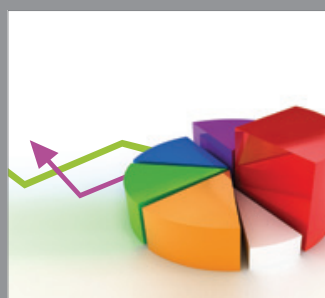

ournal of

Probability and Statistics

Promensencen
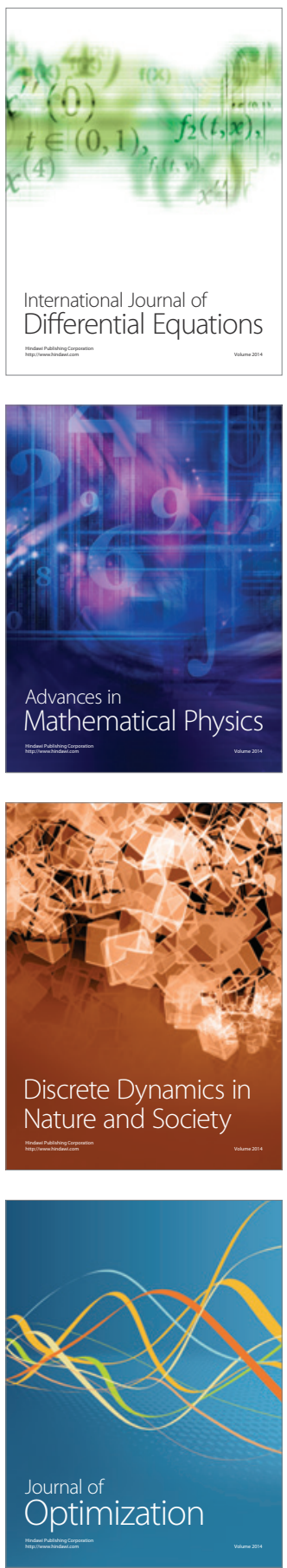